\title{
Planetary-wave-induced transport in the stratosphere
}

\section{Article}

Published Version

Pendlebury, D. and Shepherd, T. G. (2003) Planetary-waveinduced transport in the stratosphere. Journal of the Atmospheric Sciences, 60 (11). pp. 1456-1470. ISSN 15200469 doi: https://doi.org/10.1175/15200469(2003)060<1456:PTITS>2.0.CO;2 Available at https://centaur.reading.ac.uk/32120/

It is advisable to refer to the publisher's version if you intend to cite from the work. See Guidance on citing.

To link to this article DOI: http://dx.doi.org/10.1175/1520-

0469(2003)060<1456:PTITS>2.0.CO;2

Publisher: American Meteorological Society

All outputs in CentAUR are protected by Intellectual Property Rights law, including copyright law. Copyright and IPR is retained by the creators or other copyright holders. Terms and conditions for use of this material are defined in the End User Agreement.

\section{www.reading.ac.uk/centaur}

\section{CentAUR}


Central Archive at the University of Reading

Reading's research outputs online 


\title{
Planetary-Wave-Induced Transport in the Stratosphere
}

\author{
Diane Pendlebury* and Theodore G. Shepherd \\ Department of Physics, University of Toronto, Toronto, Ontario, Canada
}

(Manuscript received 7 January 2002, in final form 19 December 2002)

\begin{abstract}
In the stratosphere, chemical tracers are drawn systematically from the equator to the pole. This observed Brewer-Dobson circulation is driven by wave drag, which in the stratosphere arises mainly from the breaking and dissipation of planetary-scale Rossby waves. While the overall sense of the circulation follows from fundamental physical principles, a quantitative theoretical understanding of the connection between wave drag and Lagrangian transport is limited to linear, small-amplitude waves. However, planetary waves in the stratosphere generally grow to a large amplitude and break in a strongly nonlinear fashion. This paper addresses the connection between stratospheric wave drag and Lagrangian transport in the presence of strong nonlinearity, using a mechanistic three-dimensional primitive equations model together with offline particle advection. Attention is deliberately focused on a weak forcing regime, such that sudden warmings do not occur and a quasi-steady state is reached, in order to examine this question in the cleanest possible context.

Wave drag is directly linked to the transformed Eulerian mean (TEM) circulation, which is often used as a surrogate for mean Lagrangian motion. The results show that the correspondence between the TEM and mean Lagrangian velocities is quantitatively excellent in regions of linear, nonbreaking waves (i.e., outside the surf zone), where streamlines are not closed. Within the surf zone, where streamlines are closed and meridional particle displacements are large, the agreement between the vertical components of the two velocity fields is still remarkably good, especially wherever particle paths are coherent so that diabatic dispersion is minimized. However, in this region the meridional mean Lagrangian velocity bears little relation to the meridional TEM velocity, and reflects more the kinematics of mixing within and across the edges of the surf zone. The results from the mechanistic model are compared with those from the Canadian Middle Atmosphere Model to test the robustness of the conclusions.
\end{abstract}

\section{Introduction}

Observations of long-lived tracers, such as $\mathrm{N}_{2} \mathrm{O}$ (Randel et al. 1993), reflect the mean meridional mass circulation in the stratosphere, which consists of an ascending branch in the Tropics and summer subtropics, and descending branches at the poles. The overturning time of the stratosphere via this so-called Brewer-Dobson circulation is approximately $5 \mathrm{yr}$ (Rosenlof 1995). The mean meridional circulation is constrained by the dual requirements that diabatic heating/cooling of air is needed for persistent vertical motion, and an applied torque is needed for persistent meridional motion. Because of the relaxational nature of radiative heating/ cooling in the stratosphere, the circulation is in fact driven by the applied torque, and the diabatic heating/ cooling arises in response to the circulation (Fels 1985;

\footnotetext{
* Current affiliation: NorthWest Research Associates, Bellevue, Washington.
}

Corresponding author address: T. G. Shepherd, Dept. of Physics, University of Toronto, 60 St. George Street, Toronto, ON, M5S 1A7, Canada.

E-mail: tgs@atmosp.physics.utoronto.ca
Haynes et al. 1991). Exceptions are the transient circulation associated with the seasonal cycle (Garcia 1987), and the solstitial middle atmosphere Hadley circulation (Dunkerton 1989; Plumb and Eluszkiewicz 1999; Semeniuk and Shepherd 2001b).

The applied torque that drives the Brewer-Dobson circulation arises mainly from planetary-scale Rossby waves, which are generated in the troposphere and propagate into the middle atmosphere where they grow in amplitude, break, and dissipate. (The stratospheric circulation is also affected by gravity wave drag, but this is a secondary contribution.) Dissipating planetary waves apply a negative torque, and so produce a poleward mass transport at midlatitudes (i.e., toward the earth's axis of rotation, and therefore to smaller values of angular momentum), resulting in upwelling in the Tropics and downwelling at the poles through mass continuity (see e.g., Shepherd 2000, 2002). Since the forced Rossby waves tend to be quasi stationary and therefore may only propagate in the winter westerlies (Charney and Drazin 1961), there is a seasonal dependence to the Brewer-Dobson circulation. As a result, the poleward circulation occurs mainly in the winter hemisphere, with only a weak poleward cell confined to the lowermost stratosphere in the summer hemisphere. 
The current picture of stratospheric transport is largely based on the transformed Eulerian mean (TEM) equations, as described by Andrews et al. (1987). This framework recognizes the significant role that eddies play in transport, in such a way that the applied torque due to angular momentum transfer by eddies is concisely expressed in the Eliassen-Palm flux divergence (EPFD). This torque drives a meridional circulation known as the TEM or residual circulation, which is essentially the mean mass flux. However, the connection between the TEM circulation and the mean motion of air parcels is not self-evident. In the special case of the linearized equations, under steady and conservative conditions within the quasigeostrophic approximation, it can be shown that the TEM circulation is equal to the mean Lagrangian circulation (Dunkerton 1978; Rood and Schoeberl 1983; Mo and McIntyre 1997). However, under these conditions the Charney-Drazin nonacceleration theorem states that the TEM circulation vanishes (Edmon et al. 1980).

The condition of quasigeostrophy is not fundamental to the nonacceleration theorem (Andrews 1983), and the condition of steadiness is not unreasonable over a seasonal cycle (Randel et al. 2002). However, the conditions of linear and conservative dynamics are definitely not relevant in the stratosphere; radiative damping is required to allow the Brewer-Dobson circulation, and the process of planetary wave dissipation through wave breaking, which drives the Brewer-Dobson circulation, is highly nonlinear. Planetary wave breaking is also responsible for producing the surf zone where chemical constituents are well mixed along isentropic surfaces (McIntyre and Palmer 1983; Offermann et al. 1999; Ngan and Shepherd 1999). Thus, the quantitative connection between the TEM circulation and Lagrangian mean motion remains to be established under more realistic conditions.

Other methods of determining the mean mass transport have been used. A hybrid Eulerian-Lagrangian circulation known as the generalized Lagrangian mean (GLM; Andrews and McIntyre 1978) has been used to extend the theory of Lagrangian mean motion to finite amplitude. However, computational problems exist with the GLM (McIntyre 1980a). More fundamentally, while the GLM seems well designed to handle finite-amplitude but nonbreaking waves, in regions of breaking waves where Lagrangian trajectories may become chaotic, the material contours over which averages are calculated in the GLM framework become space-filling and uncomputable in the long-time limit. This is a serious problem for analysis in the stratospheric surf zone.

Another proposed method is the modified Lagrangian mean (MLM; McIntyre 1980b; Nakamura 1995), which uses the area enclosed by contours of long-lived tracers on isentropic surfaces to diagnose transport. For that reason it is a tracer-specific diagnostic, although similar tracers lead to similar results. The circulation obtained from the MLM has advantages over that from the GLM since the resulting velocities obey a continuity equation. However, because long-lived tracers tend to become highly convoluted, especially in the surf zone, the MLM is difficult to apply directly to the tracer fields themselves and the most successful application has been to an artificially generated tracer field of finite lifetime (Haynes and Shuckburgh 2000). The MLM has also been used for instantaneous calculations of equivalent length (Nakamura and Ma 1997; Allen et al. 1999) to diagnose transport barriers and regions of mixing, but the circulation diagnosed using the MLM has not been directly compared with the TEM circulation. A comparison of MLM and TEM is warranted, but not given here.

It remains the case that the TEM circulation has a clear physical connection to wave-drag driving, and is relatively easy to compute from stratospheric data. Thus the quantitative connection between the TEM circulation and mean Lagrangian motion is of interest; this connection is the focus of the present paper. Previous studies of the stratospheric wave-induced Lagrangian circulation have been analytical and linear (e.g., Schoeberl 1981; Rood and Schoeberl 1983; Mo and McIntyre 1997), with mechanical dissipation represented by Rayleigh friction. In order to study the Brewer-Dobson circulation in a more realistic context, it is necessary to use a three-dimensional model with fully nonlinear and interacting waves, where the mechanical dissipation occurs via small-scale diffusion following an enstrophy cascade. In this case an analytical treatment is not feasible. Instead, a mechanistic primitive equations model (PEM) is used here, which includes only adiabatic dynamics together with very simple nonconservative processes whose nature and consequences for the mean flow can be easily understood.

Section 2 provides a brief description of the PEM, its initial conditions, and the forcing mechanisms used. The model response to the planetary wave forcing is described insofar as it relates to issues of transport and mixing. In section 3, the mean Lagrangian circulation of the model is examined through the use of a threedimensional particle advection scheme. The degree to which the TEM circulation accurately describes the mean Lagrangian circulation is assessed. In section 4, results from the Canadian Middle Atmosphere Model (CMAM), a comprehensive middle atmosphere general circulation model, are studied and compared with the PEM results, to test the robustness of the conclusions under yet more realistic conditions. The paper concludes with a discussion in section 5 .

\section{Mechanistic model characterization and experiments}

\section{a. Model description}

The PEM solves the hydrostatic primitive equations for a dry, rotating, spherical atmosphere (Andrews et 
al. 1987) and was written by R. Saravanan. These equations allow for full wave-mean-flow interaction, needed in order to study the nonlinear dynamics of the BrewerDobson circulation, but the model includes only simple, well-understood dissipation processes. A horizontal spectral truncation of 42 meridional wavenumbers and 10 zonal wavenumbers is used. The relatively coarse resolution in the zonal direction is chosen because in the presence of a zonal shear flow, small scales develop preferentially in the meridional direction (Haynes and McIntyre 1987; Shepherd 1987). The vertical discretization uses 60 pressure levels spaced almost equally in log-pressure height with a resolution of $\sim 1.25 \mathrm{~km}$ in the interior of the model. The high vertical resolution is important to allow for the development of inertial instability, which will be seen to have consequences for the circulation in the Tropics. The upper and lower boundaries are at 0.005 and $200 \mathrm{mb}$, respectively.

Numerical dissipation in the model is included in the form of $\nabla^{8}$ hyperdiffusion, which preferentially damps the smallest horizontal scales, with a damping timescale of $6 \mathrm{~h}$ at spherical harmonic index $n=42$. Vertical viscosity is also used to control small vertical scales, with a damping timescale of 10 days for the smallest vertical scales except where noted. Forcing included in the model is in the form of Newtonian cooling, which relaxes the temperature back to the specified initial temperature, and an optional Rayleigh friction. Rayleigh friction, although unphysical (e.g., Shepherd et al. 1996), is considered in some runs here for continuity with previous work. When active, the Rayleigh friction acts only on the waves so that the zonal mean drag arises overwhelmingly from resolved wave drag. A separate Rayleigh-friction sponge layer is used above $70 \mathrm{~km}$ in order to dissipate waves in this region and prevent reflection off the upper boundary. Again, there is no sponge-layer friction applied on the zonal-mean winds in order to avoid spurious feedbacks from the sponge layer (Shepherd et al. 1996). Nonconservative processes act only on deviations from the initial conditions, and thus, in the absence of forced planetary waves, the model will not deviate from its initial conditions, which are dynamically stable.

The model is run in a perpetual January state and is initially set to have zero meridional and vertical winds, and a zonal wind (shown in Fig. 1) typical of a Northern Hemisphere $(\mathrm{NH})$ winter solstice. The potential temperature field is calculated using gradient wind balance. In order to keep the dynamics as simple as possible, the globally averaged vertical stratification is chosen to be that of an isothermal state with a scale height of $7 \mathrm{~km}$, which yields a squared buoyancy frequency $N^{2}$ of $4 \times$ $10^{-4} \mathrm{~s}^{-2}$. Because the focus is on the extratropics, there is no attempt to treat equatorial processes realistically. In particular, large-scale equatorial waves are not forced, which would provide zonal-mean momentum forcing at the equator. Neither is there any representation of the solstitial middle atmosphere Hadley circulation. Con-
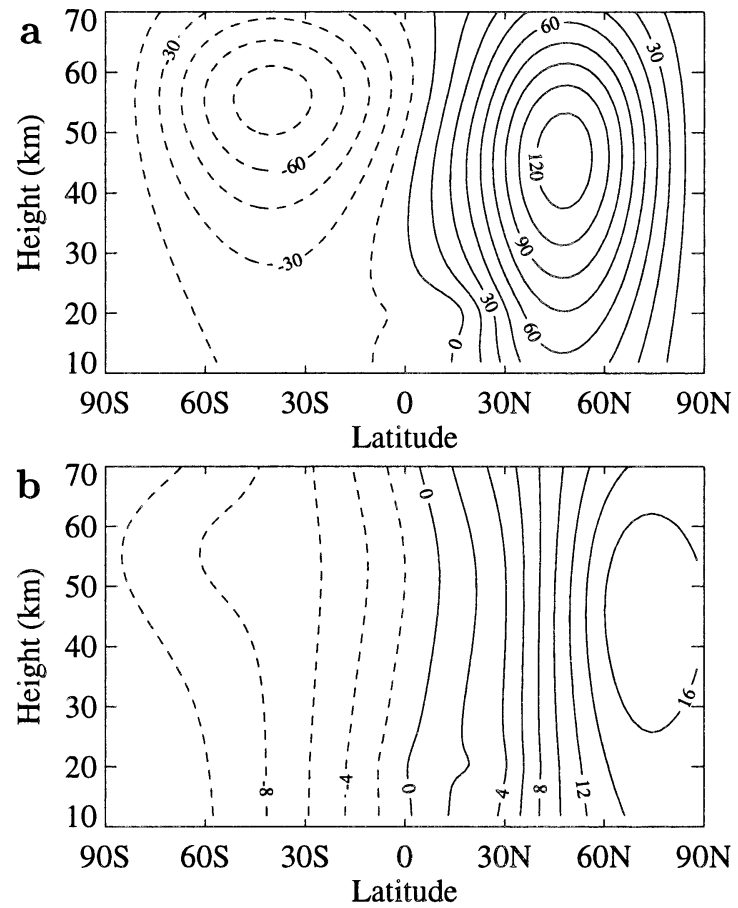

FIG. 1. The initial background fields for the runs. Shown are (a) the chosen zonal wind with contour intervals of $15 \mathrm{~m} \mathrm{~s}^{-1}$, and (b) the absolute vorticity field corresponding to the chosen zonal wind field with contour intervals of 2 day $^{-1}$. Height refers to the logpressure height defined by using a scale height of $7 \mathrm{~km}$.

sequently, the flow in the Tropics is not expected to resemble observations.

Since the model has no troposphere, upward-propagating planetary waves are generated artificially at the lower boundary. This is done by specifying the geopotential height on the lower boundary $\Phi_{L}$ to be that needed to balance the initial zonal wind, plus a term that forces planetary-scale waves in the $\mathrm{NH}$ :

$$
\Phi_{s}(\lambda, \varphi, t)=g A_{0} \cos (m \lambda) f(t) h(\varphi),
$$

where $g$ is gravitational acceleration, $A_{0}$ is the maximum forcing amplitude for each case,

$f(t)= \begin{cases}\frac{1}{2}\left\{1+\tanh \left[2\left(\frac{t}{5 \text { days }}-1\right)\right]\right\} & \text { if } t \leq 10 \text { days, } \\ 1 & \text { otherwise, }\end{cases}$

which ramps up the forcing over 10 days, and

$$
h(\varphi)= \begin{cases}(17 / 2) \hat{\mu}^{2}\left(1-\hat{\mu}^{2}\right) \cos ^{2} \varphi & \text { if } \mu \geq \hat{\mu}, \\ 0 & \text { otherwise, }\end{cases}
$$

where $\mu=\sin \varphi, \hat{\mu}=\left(\mu-\mu_{0}\right) /\left(1-\mu_{0}\right)$ and $\mu_{0}=$ $\sin (\pi / 12)$, so that the maximum forcing amplitude is at $\sim 45^{\circ} \mathrm{N}$ and $\Phi_{s}$ is zero south of $15^{\circ} \mathrm{N}$.

It is possible in this model to simulate sudden warmings and vacillation cycles (Scott and Haynes 2000; D. 
TABLE 1. Forcing and dissipation parameters.

\begin{tabular}{cccccc}
\hline \hline Run & $m$ & $A_{0}(\mathrm{~m})$ & $\begin{array}{c}\text { Rayleigh } \\
\text { friction } \\
\text { timescale } \\
\text { (days) }\end{array}$ & $\begin{array}{c}\text { Newtonian } \\
\text { cooling } \\
\text { timescale } \\
\text { (days) }\end{array}$ & $\begin{array}{c}\text { Length of } \\
\text { run (days) }\end{array}$ \\
\hline A1 & 2 & 56 & 10 & 10 & 300 \\
A2 & 2 & 94 & 10 & 10 & 240 \\
B1 & 1 & 94 & $\infty$ & 10 & 300 \\
B2 & 2 & 70 & $\infty$ & 10 & 300 \\
\hline
\end{tabular}

Ortland 2001, personal communication). However, the purpose of this study is to examine the Brewer-Dobson circulation in a quasi-steady state, and not in these more complicated situations, in order to test the relationship between the TEM and Lagrangian mean velocities in the simplest realistic context. Sudden warmings and vacillation cycles were avoided in these runs primarily by choosing a small forcing amplitude. In Scott and Haynes (2000), the transition between runs that produced steady states and those that produced vacillation cycles was a forcing amplitude of $\sim 90-130 \mathrm{~m}$ depending on the radiative basic state. Here, the maximum forcing amplitude used is $94 \mathrm{~m}$. As a consequence, the winter hemisphere jet is very strong compared with $\mathrm{NH}$ observations, and more representative of the Southern Hemisphere (SH).

\section{b. Model response}

Results are shown from four different cases. The forcing parameters and the length of each run are summarized in Table 1. Runs labeled A1 and A2 have Rayleigh friction active in the interior of the domain, while those labeled B1 and B2 have no Rayleigh friction in the interior. In the first case (A1), the planetary wave forcing is sufficiently small that the model response is only weakly nonlinear; that is to say, while nonlinear effects still contribute to the mean-flow response to the wave forcing, particularly in the early development of the run, most of the behavior is as expected from linear theory. Wave breaking is suppressed by the small amplitude of the forcing and by the Rayleigh friction, which acts directly on the forced wave. The second case (A2) still has direct mechanical damping of the forced planetary wave; however, the amplitude of the forced wave is much larger. The larger amplitude results in the development of inertial instability in the Tropics (O'Sullivan and Hitchman 1992), suggesting that nonlinear effects are more important in this region. The vertical viscosity in this case is increased by reducing the timescale to 7 days, in order to better control the inertial instability. In cases B1 and B2, the Rayleigh friction is removed so that the principal direct damping of the waves is thermal, from Newtonian cooling on the temperature perturbation, and the only damping on the wave vorticity and divergence fields is through the scale-selective horizontal and vertical diffusion. This allows the forced waves to break and cascade their enstrophy to smaller scales, so that the wave drag is accomplished in a fully nonlinear and therefore more realistic fashion.

A quasi-steady state is achieved in all runs, although the transience has not completely disappeared at the equator or near the top of the model (in the sponge layer). In each case, the waves are dissipated in the vicinity of the zero-wind line, which is a critical line. The effect of the negative wave-induced zonal force is to decelerate the zonal wind and therefore erode the equatorward side of the $\mathrm{NH}$ jet. Associated with the increase of meridional shear is a surf zone, manifested in the homogenization of absolute vorticity (and therefore of potential vorticity) from $\sim 5^{\circ}-30^{\circ} \mathrm{N}$, and the formation of a strong meridional gradient of absolute vorticity on both sides of this region (see Fig. 2). In all cases, the wave forcing extends to the equator, which allows a cross-equatorial flow to develop through nonlinear processes and inertial adjustment (Dunkerton 1991; Semeniuk and Shepherd 2001a). In the tropical regions of the PEM, the diabatic heating, and therefore the upwelling, is symmetric about the equator since 1) a nonzero meridional temperature gradient at the equator would violate gradient-wind balance, and 2) the initial temperature is symmetric about the equator. The development of a northward flow in the $\mathrm{SH}$ also produces a deceleration of the zonal wind, increasing the strength of the westward jet. In the long-time limit, dissipative processes are necessary in order to maintain a crossequatorial flow and still keep the angular momentum maximum over the equator (as is required for inertial stability).

The TEM streamfunction for each case, shown in Fig. 3 , reveals the expected Brewer-Dobson circulation. The circulation does not extend to the pole since the EPFD is concentrated on the critical line at approximately $\sim 15^{\circ}-20^{\circ} \mathrm{N}$ (the middle of the surf zone). The differences between the Eulerian mean circulation (not shown) and the TEM circulation in both runs A1 and A2 are largely restricted to north of the zero-wind line, since the waves are restricted to this region. However, in runs without Rayleigh friction (B1 and B2), the lack of direct dissipation on the larger-scale waves allows for the development of smaller-scale waves. These waves may penetrate the $\mathrm{SH}$ where they produce a nonzero EPFD. In run B2, the TEM circulation above 45 $\mathrm{km}$ is reversed from the expected response. This is due in part to some lingering transience in $\partial \bar{u} / \partial t$ over the equator, but is largely due to regions of positive EPFD on either side of the equator at this altitude.

\section{Lagrangian circulation}

Lagrangian velocities are calculated by advecting particles in isentropic coordinates. The isentropic motion is computed using a contour advection code developed by W. A. Norton and D. G. Dritschel (Dritschel 1989) with the contour surgery removed, while the cross-is- 

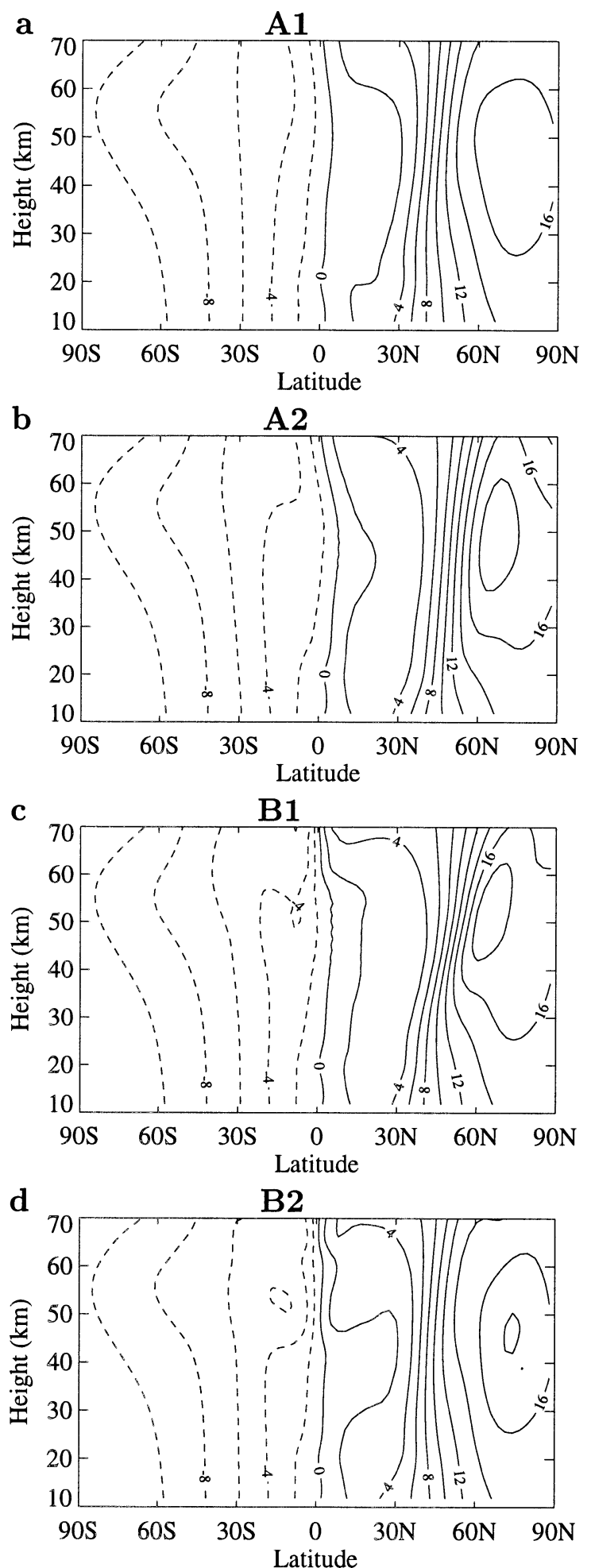

FIG. 2. The mean absolute vorticity for cases (a) A1, (b) A2, (c) B1, and (d) B2, with contour interval of 2 day $^{-1}$.
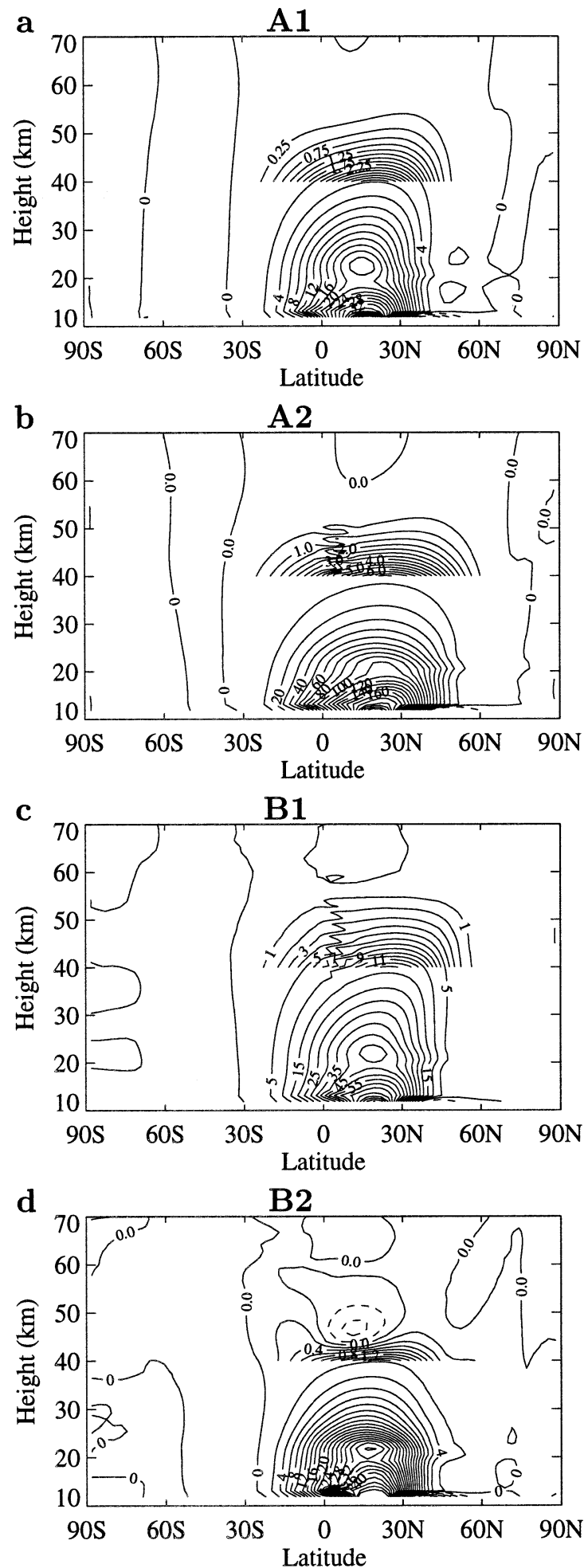

FIG. 3. As in Fig. 2 but for the TEM streamfunction. Contour intervals below and above $40 \mathrm{~km}$ are (a) 2 and $0.25 \mathrm{~kg} \mathrm{~m}^{-1} \mathrm{~s}^{-1}$, (b) 10 and $0.5 \mathrm{~kg} \mathrm{~m}^{-1} \mathrm{~s}^{-1}$, (c) 5 and $1 \mathrm{~kg} \mathrm{~m}^{-1} \mathrm{~s}^{-1}$, and (d) 2 and 0.2 $\mathrm{kg} \mathrm{m}^{-1} \mathrm{~s}^{-1}$. Positive values denote a circulation in the clockwise sense. 
a

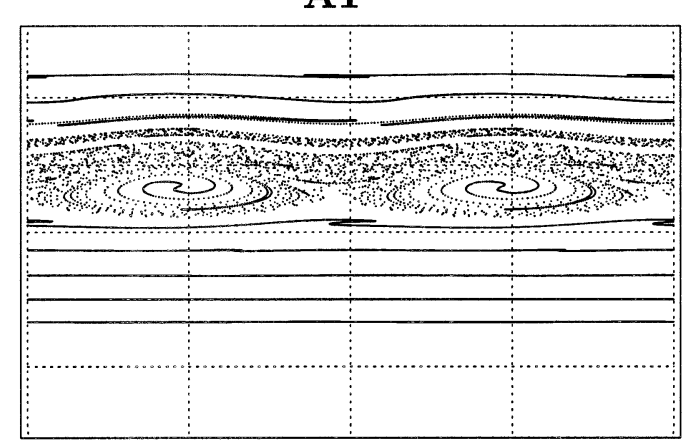

c

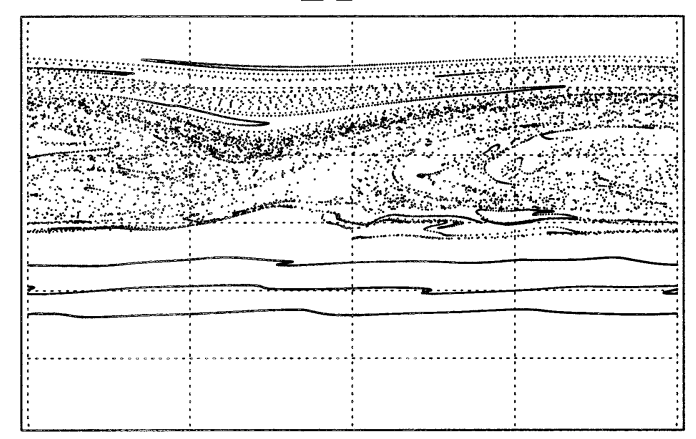

b

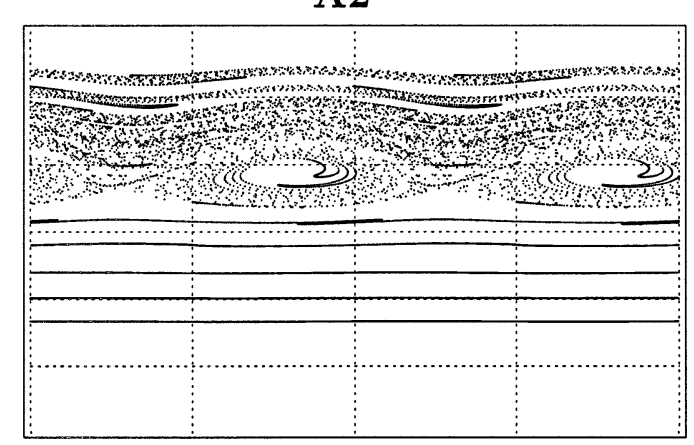

d

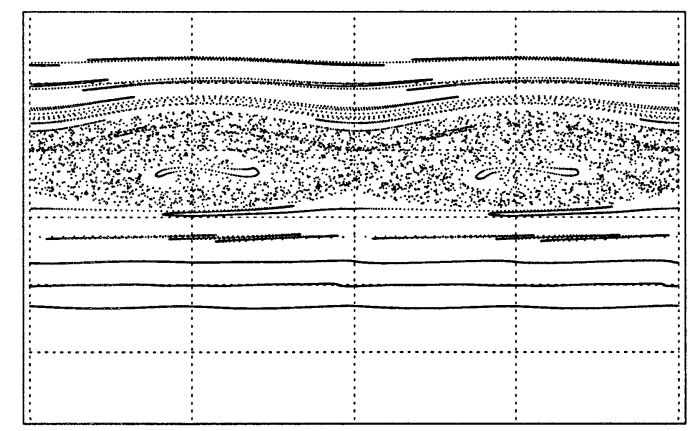

Fig. 4. Isentropic 2D particle advection for day 60 for the PEM cases (a) A1 at $1000 \mathrm{~K}$, (b) A2 at $1000 \mathrm{~K}$, (c) B1 at $1000 \mathrm{~K}$, and (d) B2 at $700 \mathrm{~K}$. The horizontal axis is longitude from $180^{\circ} \mathrm{W}$ to $180^{\circ} \mathrm{E}$, and the vertical axis is latitude from $90^{\circ} \mathrm{S}$ to $90^{\circ} \mathrm{N}$.

entropic motion is computed directly from the model diabatic heating rates. The model winds and heating rates from each run are saved every $12 \mathrm{~h}$ for the same 60 -day time period as the Eulerian diagnostic analysis, and are projected onto isentropic surfaces. The particles are then advected using a 30-min time step, and linear interpolation in $(\lambda, \varphi, \theta, t)$ space is used to calculate $u$, $v$, and $\dot{\theta}$ for each particle. The coarse spatial and temporal resolution used in the PEM to sample the winds and heating rates is sufficient for this stratospheric regime, where small-scale motion and filamentation are determined by the large-scale flow. This would not be the case in the real mesosphere where the role of gravity waves becomes important (Shepherd et al. 2000).

Particles are originally placed on constant $\theta$ surfaces and on constant latitude circles, and then allowed to evolve for 10-15 days to a more uniform distribution before the transport calculations begin. The reason for this is that otherwise the initial particle motion on an isentropic surface creates the appearance of dispersion about the latitude circle. This motion does not indicate irreversibility but merely reflects the motion of particles along different potential vorticity contours, which undulate in latitude. The effect is particularly large in the surf zone where potential vorticity is more homogenized so that particles initially placed at one latitude may travel across the width of the surf zone. The particle trajectories are complemented by backward trajectories in an attempt to smooth out some of the statistical fluctuations and isolate advective motion, as discussed in Kida (1983). Although this method did not reduce the dispersion, it was helpful in locating particles at more latitudes.

The purely adiabatic particle advection on one isentropic surface is shown in Fig. 4 for day 60 of each run. In runs $\mathrm{A} 1$ and $\mathrm{A} 2$ (the cases with Rayleigh friction, Figs. 4a,b), the particles display a wave-2 pattern only and no other wavenumbers appear to be present, even in the surf zone. Lines of particles in the surf zone remain distinct and the surf zone is dominated by coherent stirring. In contrast, runs B1 and B2 (Figs. 4c,d) exhibit both stirring and mixing in the surf zone so that while the pattern of particles is dominated by the forcing wavenumber, smaller scales are also present.

\section{a. Adiabatic transport}

The two-dimensional results examine only the adiabatic motion on a representative isentropic surface for each run. As such, they ignore diabatic heating, and therefore vertical motion of the particles. While this is not realistic for a 60-day period, it demonstrates some important characteristics of the meridional Lagrangian velocity without the added complication of the third dimension. The adiabatic results are shown in Figs. 57 for the weakly nonlinear A1 case and the more strong- 
a

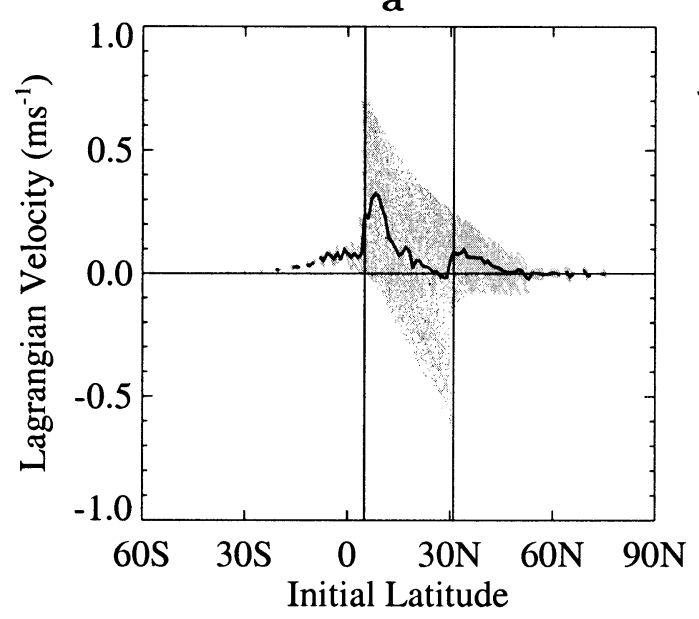

b

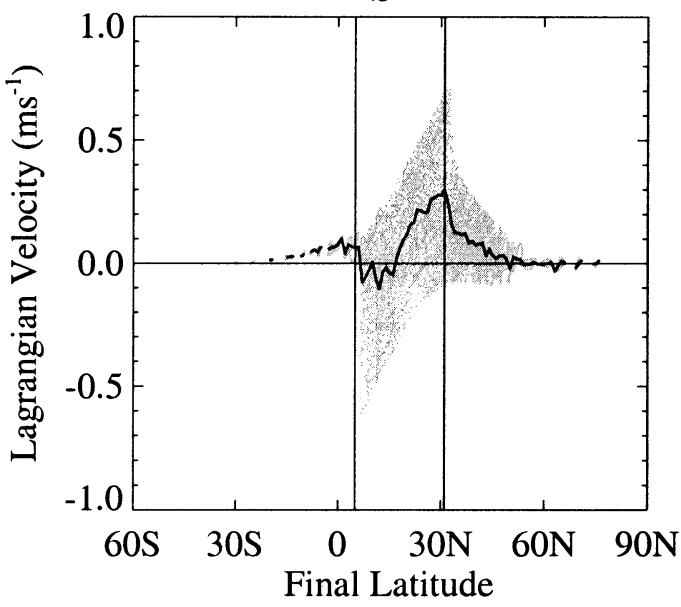

FIG. 5. Meridional Lagrangian velocity for the weakly nonlinear case A1 at the 1000-K surface using (a) initial and (b) final latitudes as particle labels. Velocities of individual particles are shown as gray dots while the zonalmean velocities of particles are shown as the black line. The position of the surf zone is indicated by the vertical lines, determined by the extent of the stirring region during the particle advection calculations. This demonstrates the bias near transport barriers resulting from the choice of particle label.

ly nonlinear B2 case. Velocities of individual particles, as defined by (4) below, are shown as gray dots, and the thick line represents the zonal-mean Lagrangian velocity. The zonal averages are calculated by binning particles in $1^{\circ}$ latitude bins and averaging each bin. Gaps in the meridional representation of the Lagrangian velocities are due to a lack of particles associated with that latitude.

The Lagrangian velocities are calculated using the portion of the particle advection calculation following the period of initialization. The time-averaged meridional and vertical Lagrangian velocities (the latter included for later reference) for each particle are calculated as

$$
v_{L}=\frac{a \pi}{180^{\circ}} \frac{\varphi_{f}-\varphi_{i}}{t_{f}-t_{i}} \text { and } Q_{L}=\frac{\theta_{f}-\theta_{i}}{t_{f}-t_{i}},
$$

where $\varphi_{i}$ and $\varphi_{f}$ are the initial and final latitudes of the particle in degrees, and $\theta_{i}$ and $\theta_{f}$ are the initial and final isentropes. However, each particle must be associated with a latitude and isentrope over the time average.

A simple choice of position label is either the initial or final position of the particle trajectory. This choice, however, can severely bias the results if the initial or final positions are very close to transport barriers, for example, very close to an edge of the surf zone. Particles that are labeled by their initial position must appear to move away from the transport barriers. Therefore, in a

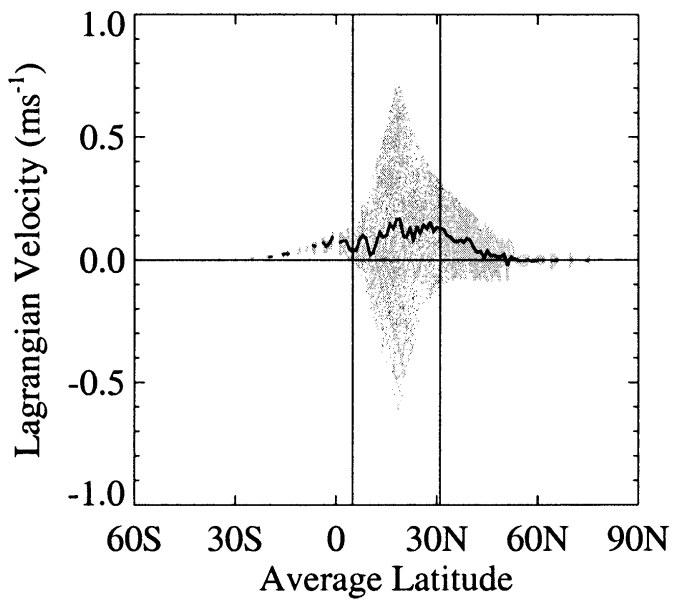

b

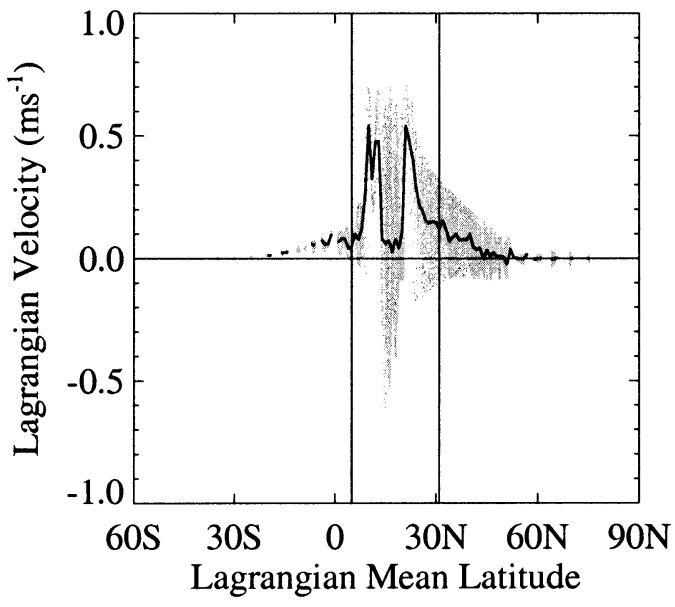

FIG. 6. As in Fig. 5 but using (a) $\varphi_{\text {avg }}$ and (b) $\varphi_{L}$ as the particle labels. 
a

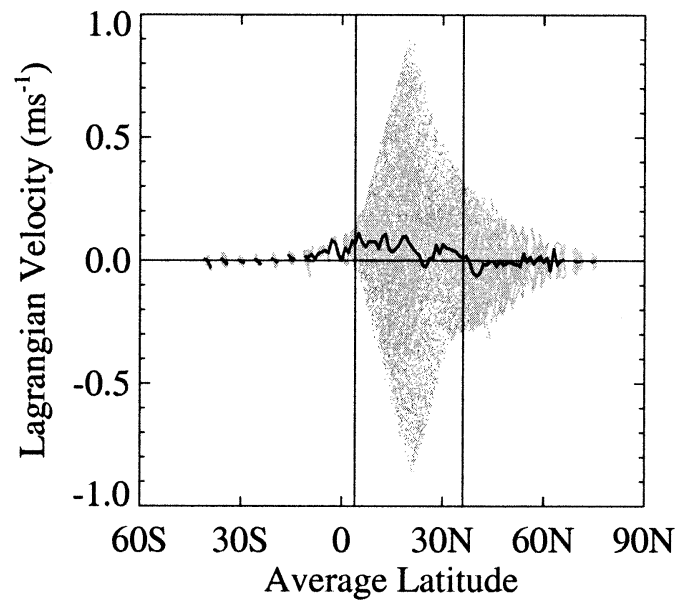

b

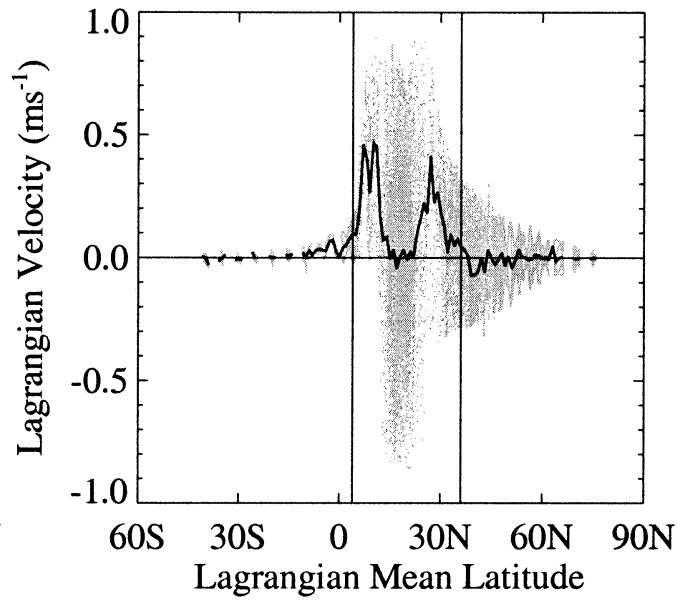

FIG. 7. As in Fig. 5 but for the strongly nonlinear case B2 at the 700-K surface using (a) $\varphi_{\text {avg }}$ and (b) $\varphi_{L}$ as the particle labels.

the surf zone, particles initially located at the southern edge of the surf zone appear to move northward, away from the edge, while particles initially located at the northern edge appear to move southward, even if the overall transport is northward. Similarly, particles labeled by their final position will appear to move toward the transport barriers. This effect is clearly shown in Fig. 5 for run A1.

An arguably more reasonable choice of particle label is to define the average latitude and isentrope, such that the particles are labeled by

$$
\varphi_{\mathrm{avg}}=\frac{\varphi_{i}+\varphi_{f}}{2} \text { and } \theta_{\mathrm{avg}}=\frac{\theta_{i}+\theta_{f}}{2} .
$$

The results are seen in Figs. 6a and 7a. The particles appear to have the widest distribution of velocities in the center of the surf zone, with this distribution decreasing almost linearly toward the edges. This is because the use of $\varphi_{\text {avg }}$ as a particle label is effectively averaging the two previous methods. Thus, particles in the surf zone will most frequently have a $\varphi_{\text {avg }}$ located in the middle of the surf zone, in which case they can exhibit relatively large velocities in either direction since the range of $\varphi_{i}$ and $\varphi_{f}$ is maximal, while those with $\varphi_{\text {avg }}$ closer to the transport barriers will necessarily exhibit less movement, since $\varphi_{i}$ and $\varphi_{f}$ are closer together. However, this method does not reflect the actual path, or time-mean position along the path of the particle.

A more physically meaningful way of labeling particles is by a Lagrangian average; that is,

$$
\varphi_{L}=\frac{1}{N} \sum_{n=1}^{N} \varphi_{n} \text { and } \theta_{L}=\frac{1}{N} \sum_{n=1}^{N} \theta_{n},
$$

where $N$ is the number of time steps considered and $\varphi_{n}$ and $\theta_{n}$ are the locations of the particles at time step $n$. In these calculations, for the purpose of labeling the particles, the time step is taken to be $12 \mathrm{~h}$. Using $\varphi_{L}$ as the particle label, the surf zone is clearly visible in the meridional velocity plot in Figs. $6 b$ and $7 b$, distinguished by the cloud of dots centered on it. This cloud is the result of particles trapped inside the surf zone, which are traveling back and forth between the two transport barriers. In a Lagrangian-averaged sense, most of the particles will be associated with the center of the surf zone, and their average meridional velocities will be either positive or negative (with nearly equal likelihood) so that the zonal average here will be a minimum. Surrounding this region, the zonal average of the Lagrangian velocities shows two peaks, also inside the surf zone. The peak near the southern edge of the surf zone is created by particles that have spent the first part of the integration south of the surf zone and then moved into the surf zone for the second part of the integration. The mean velocities of such particles are relatively large and positive, and their Lagrangian-averaged positions lie between the mean latitude of the two regions. The peak near the northern edge is created in the same way by particles that begin inside the surf zone and are transported north. Very few particles are associated with either of these two regions. These results imply a systematic northward transport; however, it is not uniform as suggested by the TEM circulation in Fig. 3. The maximum Lagrangian velocity of particles in the surf zone in either meridional direction may be obtained from the maximum spread about the zonal mean $\bar{v}_{L}$.

Outside the surf zone, both methods of labeling the particles give the same results (in fact all methods do). This is the region of nonbreaking waves where the TEM meridional velocity $\bar{v}^{*}$ is expected to agree with $\bar{v}_{L}$. As will be seen, both methods of calculating $\bar{v}_{L}$ agree very well with $\bar{v}^{*}$ in this region; however, there is another feature of interest. North of the surf zone in both cases, the Lagrangian velocity calculations using both methods 
a A1
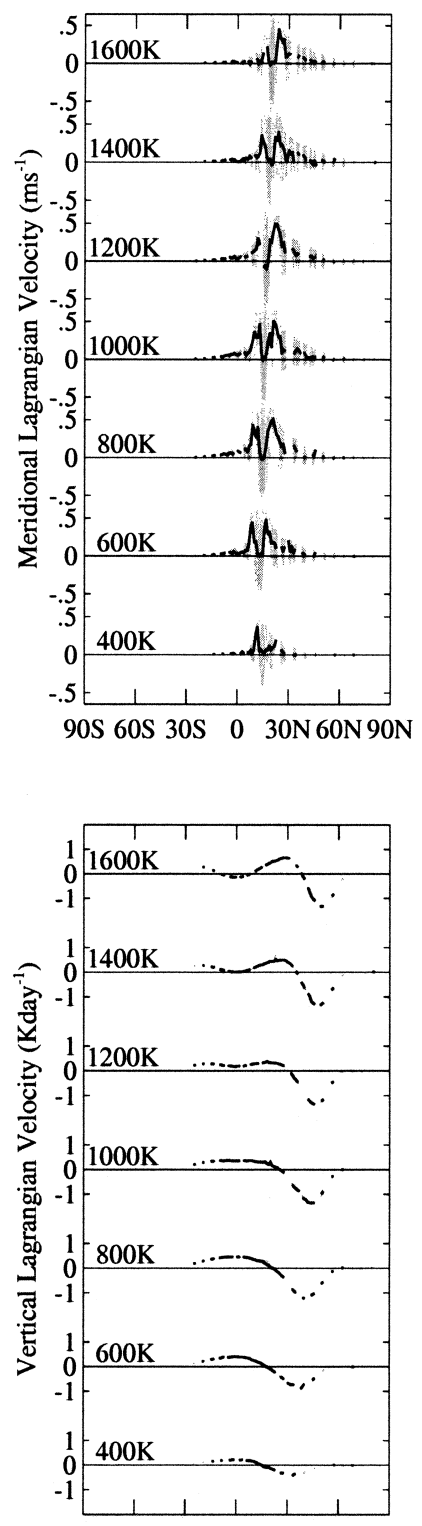

90S 60S 30S 0 30N 60N 90N b A2
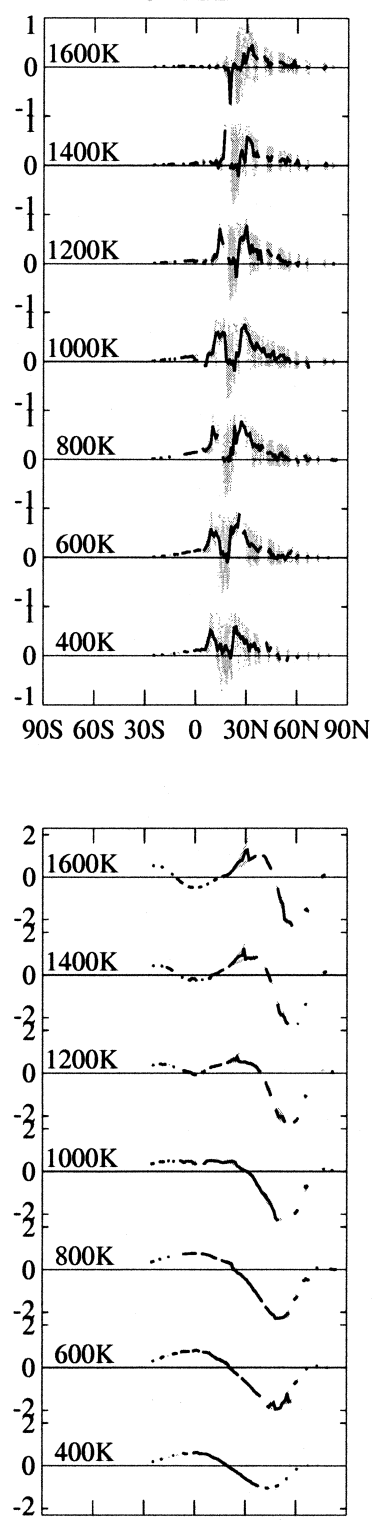

90S 60S 30S 0 30N60N90N c B1
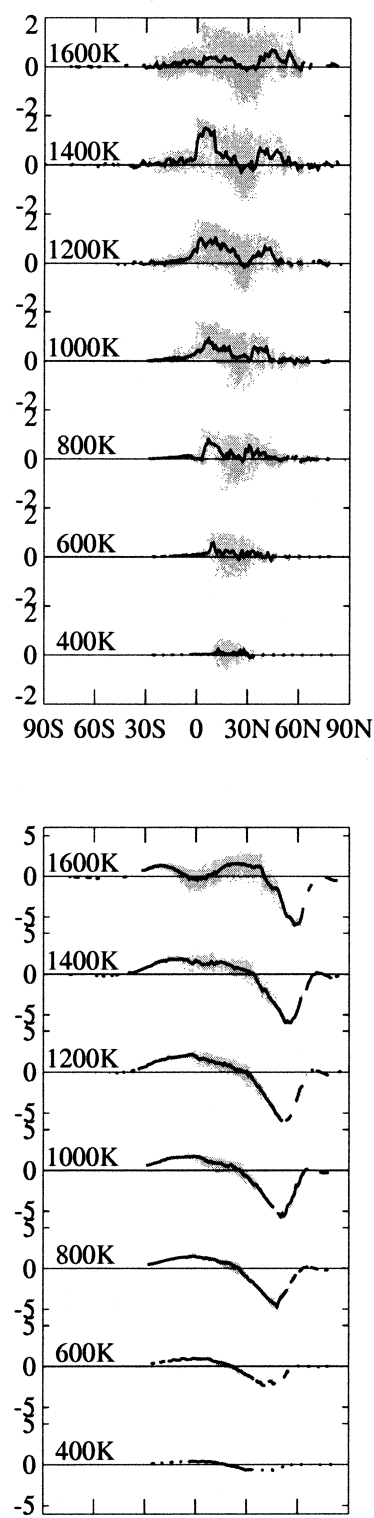

90S 60S 30S 0 30N60N 90N d B2
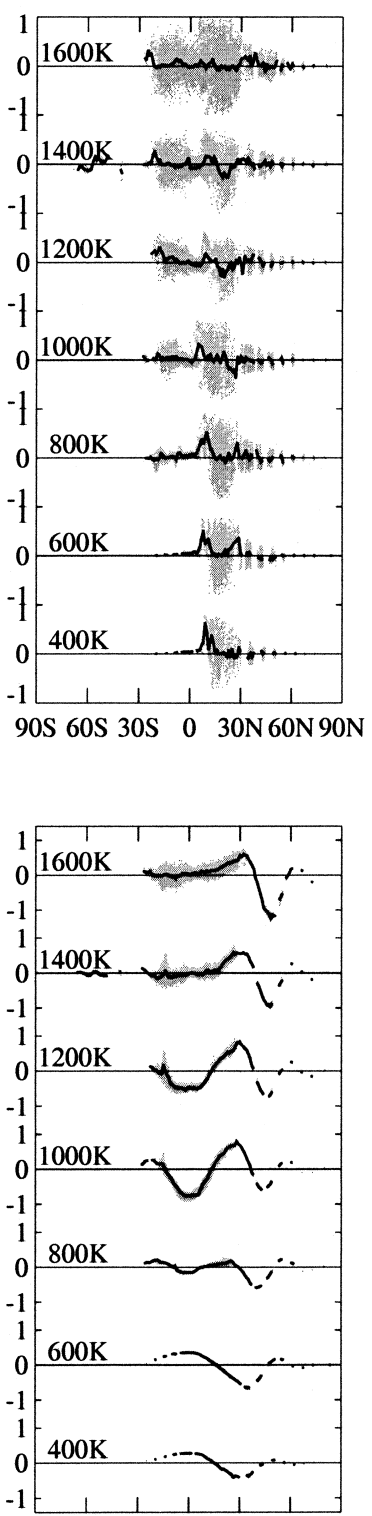

90S 60S 30S 0 30N60N 90N

FIG. 8. 3D particle advection calculations for the PEM using $\varphi_{L}$ as the particle label. Columns show runs (a) A1, (b) A2, (c) B1, and (d) B2. Rows show (top) meridional Lagrangian velocities (in $\mathrm{m} \mathrm{s}^{-1}$ ) and (bottom) vertical Lagrangian velocities (in $\mathrm{K}^{-1}{ }^{-1}$ ) on selected isentropic levels.

show a large distribution of velocities. South of the surf zone, however, the velocities show very little spreading, due to the lack of waves. Correspondingly, Fig. 4 shows very little evidence of undulating particle lines in this region. This suggests that the nature of the transport north of the surf zone is fundamentally different from that south of it. That is, transport north of the surf zone is mainly due to a wave-induced Stokes drift, while transport south of the surf zone seems to be a slow, steady, waveless drift northward, due to the nonlocal response of the mean flow to the wave forcing.

\section{b. Diabatic transport}

The three-dimensional particle advection results calculated using (4) are shown in Fig. 8 for each of the four cases. The Lagrangian-averaged positions calculated from (6) are used since they provide the truest time average of the position of a particle. The BrewerDobson circulation is clearly represented here. At every level the meridional transport is clearly poleward, except for those levels in case B2 in the region of the anomalous EPFD where the TEM circulation is reversed 
a $\mathrm{A} 1$

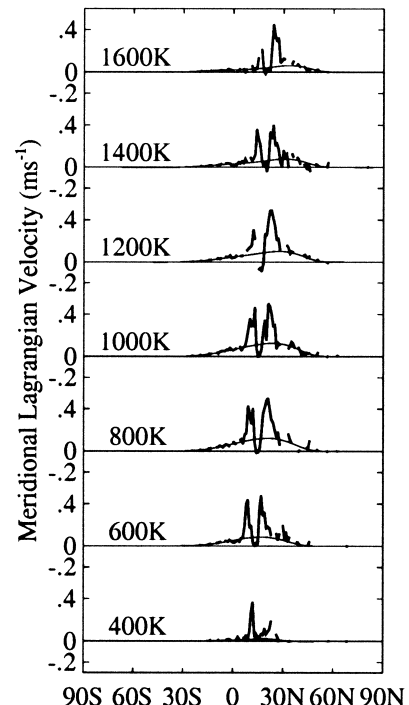

90S 60S $30 \mathrm{~S} \quad 0 \quad 30 \mathrm{~N} 60 \mathrm{~N} 90 \mathrm{~N}$

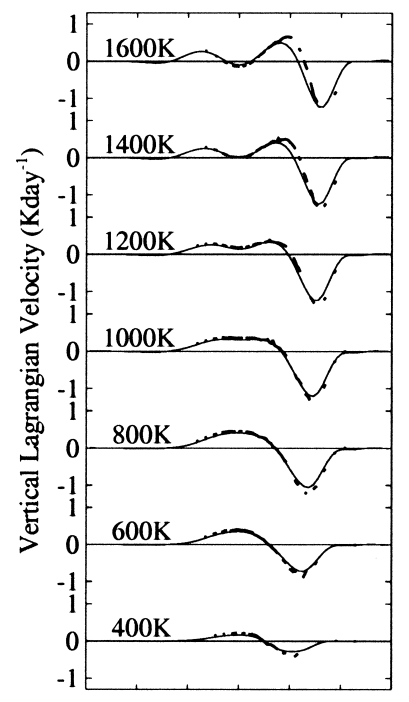

90S 60S $30 \mathrm{~S} \quad 0 \quad 30 \mathrm{~N} 60 \mathrm{~N} 90 \mathrm{~N}$ b A2
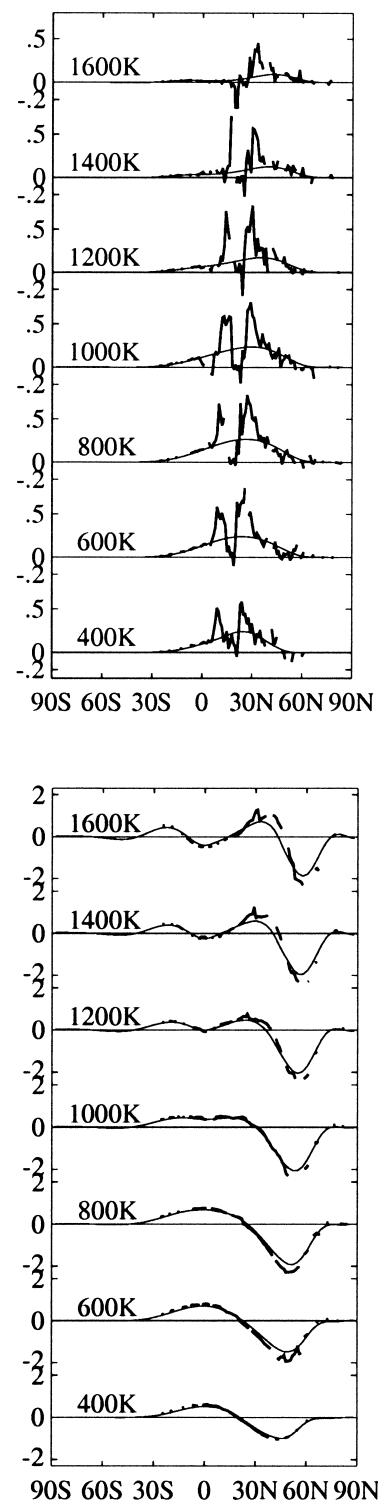

c B1

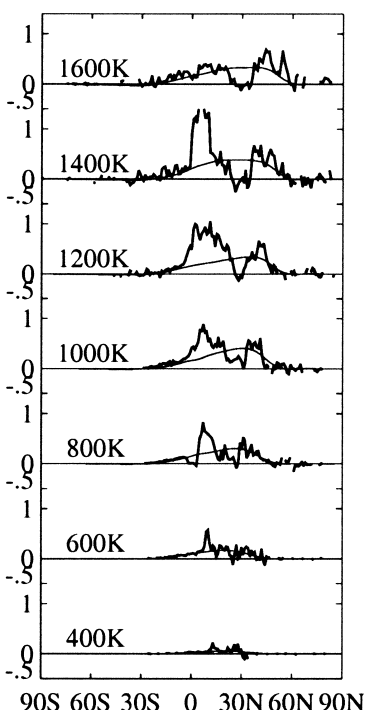

90S 60S 30S $0 \quad 30 \mathrm{~N} 60 \mathrm{~N} 90 \mathrm{~N}$

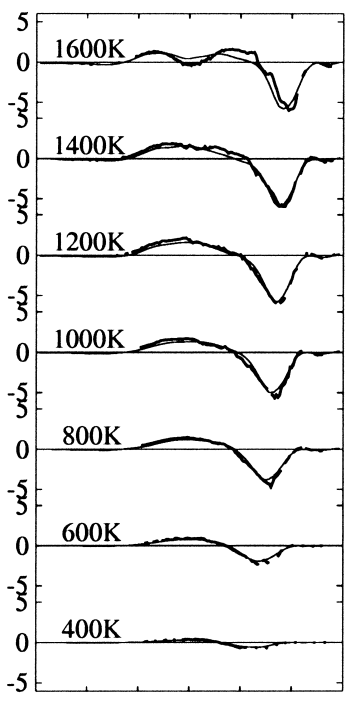

$90 \mathrm{~S} 60 \mathrm{~S} 30 \mathrm{~S} \quad 0 \quad 30 \mathrm{~N} 60 \mathrm{~N} 90 \mathrm{~N}$ d B2
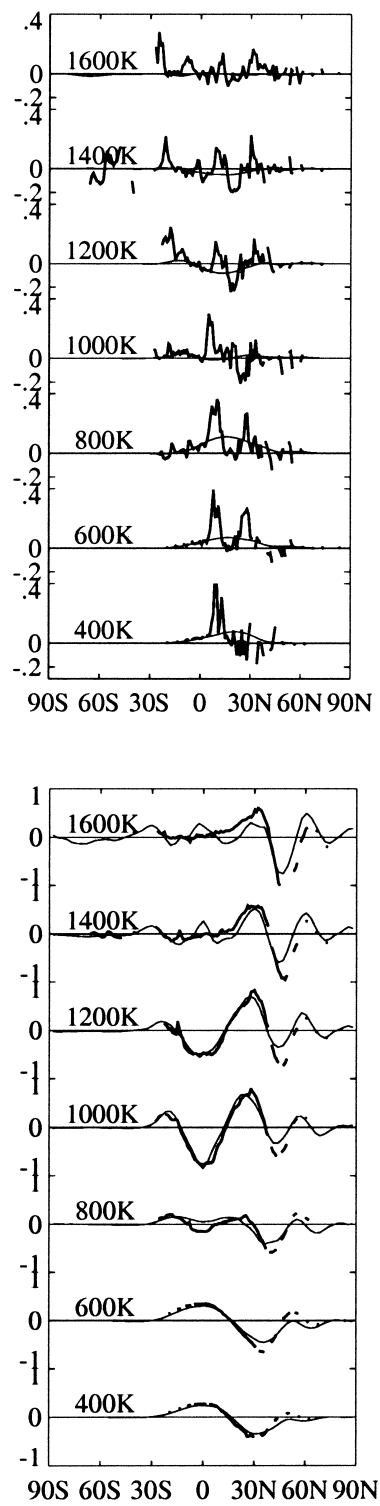

FIG. 9. Comparison of the TEM (thin line) and Lagrangian (thick line) velocities for the $3 \mathrm{D}$ particle advection calculations (using $\varphi_{L}$ as the particle label) for the PEM for cases (a) A1, (b) A2, (c) B1, and (d) B2. Rows show (top) meridional Lagrangian velocities (in $\mathrm{m} \mathrm{s}^{-1}$ ) and (bottom) vertical Lagrangian velocities (in $\mathrm{K} \mathrm{day}^{-1}$ ) on selected isentropic levels.

(above $\sim 1000 \mathrm{~K}$ ), and the vertical velocities show upwelling at the equator and downwelling at the poles. The meridional velocities were discussed in the previous section and so only differences from the features already mentioned will be discussed here.

In runs with Rayleigh friction (A1 and A2), the vertical Lagrangian velocities show very little or no spreading about the zonal mean. That is, all particles labeled by the same latitude move at the same mean vertical velocity. Outside the surf zone this is expected since material contours are not deforming irreversibly. Therefore, all particles with the same potential vorticity move along the same undulating contour, are labeled by the same mean latitude, and sample the same heating rates. Hence, there is no diabatic dispersion of particles (i.e., spreading in $\theta$ due to variations in heating rates). Within the surf zone one might expect dispersion; however, the particle advection in these cases is coherent-dominated by stirring as opposed to mixing - and so particles with the same latitude history will have the same heatingrate history. Again, the particle evolution is regular, although particle lines are wrapped up. Previous studies using GCMs (Kida 1983) or reanalyzed winds and diabatic heating rates (Sparling et al. 1997) have shown an even higher degree of diabatic dispersion in the vertical than here (see also section 4). 


\section{c. Comparison of TEM and Lagrangian velocities}

In order to compare the Lagrangian and TEM velocities, the TEM velocities from the model diagnostics are interpolated onto isentropic surfaces. Shown in Fig. 9 are the meridional TEM velocity compared with the meridional Lagrangian velocity, and the vertical TEM velocity scaled by the vertical stratification $\partial \bar{\theta} / \partial p$, denoted by $\bar{Q}^{*}$, compared with the vertical Lagrangian velocity $\bar{Q}_{L}$. Only the zonal-mean Lagrangian velocities are shown since these are the quantities of greatest interest.

In cases with Rayleigh friction (A1 and A2), where diabatic dispersion is absent, the vertical Lagrangian velocities and scaled TEM velocities compare remarkably well. There is a slight difference between the two at higher $\theta$ values, but this is where the meridional gradient of $\theta$ becomes important in the thermodynamic equation. The extent of this agreement is due to the definition of $\varphi_{L}$ and the meridional dependence of $\bar{Q}$. A Taylor expansion of $\bar{Q}^{L}$ gives

$$
\begin{aligned}
\bar{Q}^{L}= & \bar{Q}\left(\varphi_{0}\right)+\left.\frac{d \bar{Q}}{d \varphi}\right|_{\varphi_{0}}\left(\bar{\varphi}_{L}-\varphi_{0}\right) \\
& +\left.\frac{1}{2} \frac{d^{2} \bar{Q}}{d \varphi^{2}}\right|_{\varphi_{0}} \overline{\left[\left(\varphi-\varphi_{0}\right)^{2}\right]}+\cdots
\end{aligned}
$$

If the Lagrangian velocity is labeled by the Lagrangian mean latitude, then $\bar{\varphi}_{L}=\varphi_{L}=\varphi_{0}$ and the linear term in (7) vanishes. The quadratic term is small if either $\left|d^{2} \bar{Q} / d \varphi^{2}\right|$ is small, which is true inside the surf zone for these cases, or $\overline{\left[\left(\varphi-\varphi_{0}\right)^{2}\right]}$ is small, which is true outside the surf zone. Thus, $\bar{Q}_{L}$ and $\bar{Q}^{*} \approx \bar{Q}$ tend to be very similar.

As particle trajectories become more chaotic and smaller scales develop, as in cases B1 and B2, $\bar{Q}_{L}$ and $\bar{Q}^{*}$ show more differences. Although they are generally of the same sign and their maxima and minima are usually collocated, the magnitude of $\bar{Q}_{L}$ tends to be greater than that of $\bar{Q} *$ at the extrema. This difference occurs because $Q$ is zonally asymmetric at its extrema, so that taking an Eulerian mean tends to smooth out the extrema. In contrast, the Lagrangian mean is taken along particle paths and therefore potential vorticity contours, which are approximately in phase with the $Q$ contours. Nevertheless, the Lagrangian and TEM velocities still agree remarkably well.

The meridional Lagrangian velocities in this case also compare very well with the meridional TEM velocities outside the surf zone. However, inside the surf zone the Lagrangian velocities show a minimum in the middle of the surf zone and peaks on either side, as noted earlier, while the TEM velocities do not, even in the weakly nonlinear cases A1 and A2. The meridional Lagrangian velocity clearly shows nonuniform motion with latitude, and does not satisfy a continuity equation with the vertical component. In the center of the surf zone, the sys- tematic mean transport is slow, although individual particles may have large positive and negative Lagrangian velocities. Near the edges of the surf zone, the apparent motion is fast, although few particles are associated with this movement. This is an artifact of the slow movement across the transport barriers and is related to the somewhat paradoxical behavior of the Lagrangian velocity near rigid boundaries (Rhines 1977). The TEM meridional velocity does not reflect this behavior, but shows slow, steady movement poleward. The agreement worsens as the nonlinearity increases.

These comparisons were also done using average latitude $\varphi_{\text {avg }}$ as the particle label (not shown). While the meridional Lagrangian and TEM velocities then tend to agree more closely, since the peaks and the minimum in the surf zone are removed, the vertical velocities show less agreement. This can be understood from (7) in that $\bar{\varphi}_{L} \neq \varphi_{\text {avg }}=\varphi_{0}$, so then the linear term in (7) is no longer small. Here, $\bar{Q}_{L}$ takes on a stepped appearance and the velocities of individual particles show more spreading from the zonal average. Thus, while this method removes the large peaks in $\bar{v}^{L}$ that are produced by Lagrangian averaging, it is not clear that the results are a better match to the TEM velocities. For this reason, the Lagrangian-averaged latitudes are favored. There is very little difference between using $\theta_{L}$ and $\theta_{\text {avg }}$ since motion in isentropic coordinates is not wavelike; however, $\theta_{L}$ is used to be consistent.

\section{CMAM results}

While the PEM is a useful tool for studying tracer transport in a simplified stratosphere, the results are somewhat idealized. The lack of more complex physical processes, while useful for isolating effects, inhibits certain realistic behavior such as diabatic dispersion. In order to study a more realistic situation, the CMAM, which includes a troposphere and more complicated physical processes, is used. The version of the CMAM used here has T32 horizontal spectral resolution, with a corresponding physical grid of $5.6^{\circ}$ and a vertical resolution of $3 \mathrm{~km}$ in the stratosphere. Details of this version of the CMAM are documented in Beagley et al. (1997). Results are given in log-pressure coordinates, as with the PEM results. Model fields are sampled every $18 \mathrm{~h}$ in order to create the monthly averages and to collect the winds and diabatic heating rates used for the particle advection. Results are complicated by the fact that the model is not in a quasi-steady state due to the seasonal cycle.

Figure 10 shows monthly mean fields corresponding to those shown for the PEM, for one July of a CMAM run. The SH winter was chosen for this study since $\mathrm{SH}$ winters generally do not exhibit sudden warmings and tend to have a very stable polar vortex, similar to the PEM runs. Also, only the CMAM stratosphere (up to $55 \mathrm{~km}$ ) is shown here, since gravity wave drag becomes important in the mesosphere, and the Brewer-Dobson 

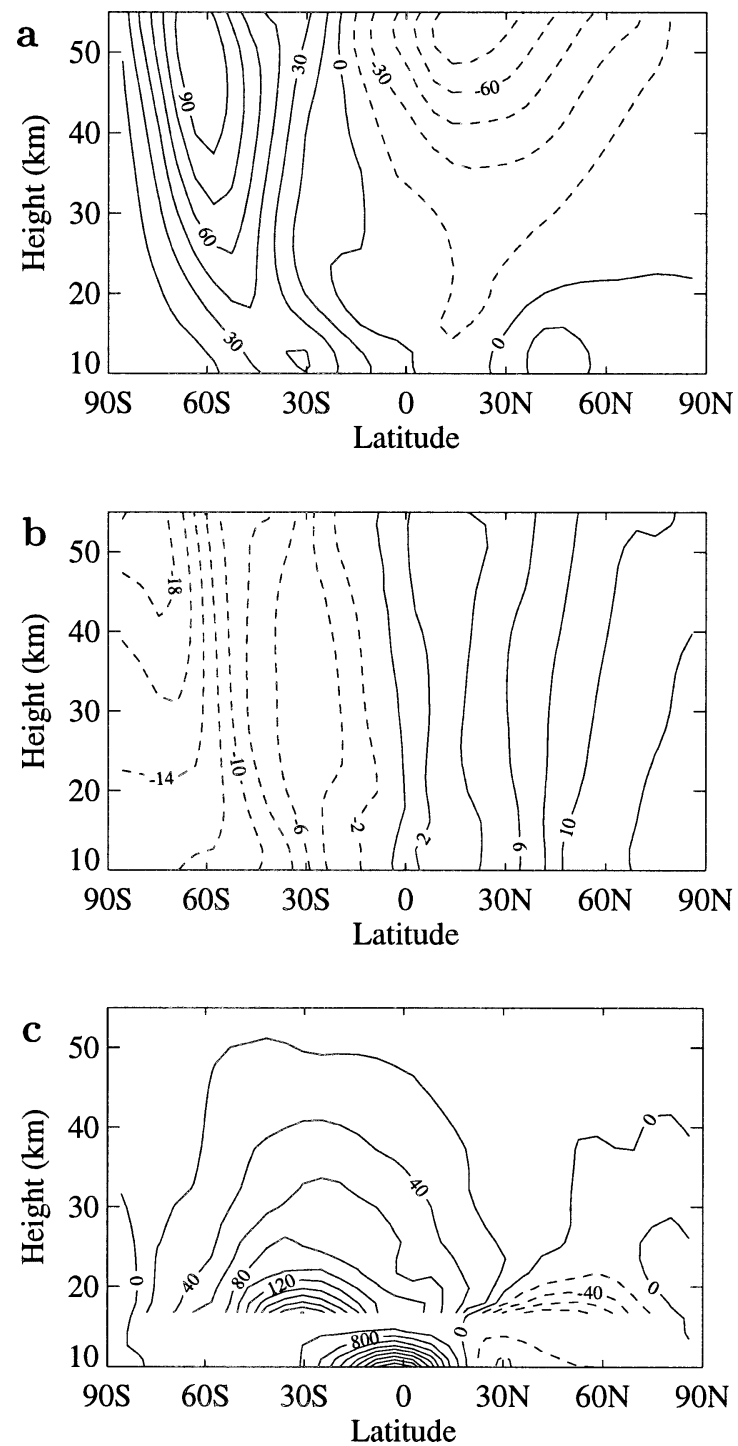

FIG. 10. Dynamical fields from a SH winter from the CMAM: (a) the zonal wind with contour interval of $15 \mathrm{~m} \mathrm{~s}^{-1}$, (b) the absolute vorticity field with contour interval $2 \mathrm{day}^{-1}$, and (c) the TEM streamfunction with contour intervals of $400 \mathrm{~kg} \mathrm{~m}^{-1} \mathrm{~s}^{-1}$ below $15 \mathrm{~km}$ and $20 \mathrm{~kg} \mathrm{~m}^{-1} \mathrm{~s}^{-1}$ above $15 \mathrm{~km}$. Positive values denote a circulation in a counterclockwise sense.

circulation is replaced by a global circulation cell from the summer to the winter hemisphere. The PEM does not contain any of the physical processes necessary to drive such a mesospheric circulation.

The TEM circulation is stronger in the CMAM than in the PEM due to the presence of more wave activity, leading to stronger EP flux convergence and wave forcing. Qualitatively, however, the circulation below $50 \mathrm{~km}$ resembles that of the PEM, particularly in the more strongly nonlinear cases (runs B1 and B2). There is also a stronger circulation in the summer hemisphere than in the PEM, although the differences between $\bar{v}$ and $\bar{v}^{*}$ are largely restricted to south of $20^{\circ} \mathrm{S}$ (the approx- a

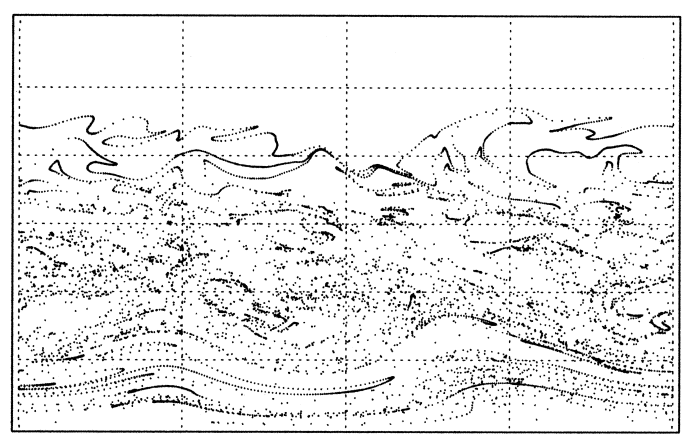

b

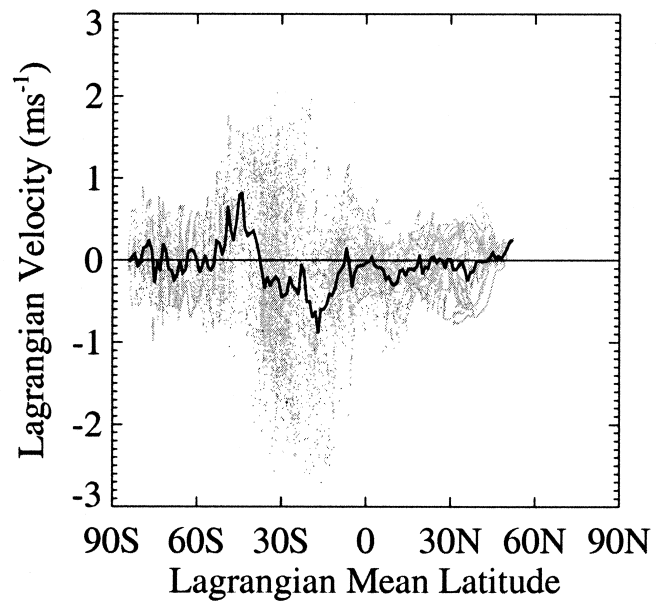

FIG. 11. (a) Adiabatic particle advection at the 1000-K isentropic surface for the CMAM run on day 30 . The horizontal axis is longitude, from $180^{\circ} \mathrm{W}$ to $180^{\circ} \mathrm{E}$, and the vertical axis is latitude, from $90^{\circ} \mathrm{S}$ to $90^{\circ} \mathrm{N}$. (b) Meridional Lagrangian velocity for the CMAM run on the $1000-\mathrm{K}$ surface using $\varphi_{L}$ as the particle label.

imate location of the zero-wind line). A region of inertial instability in the Tropics is not visible here, but it is also present (Semeniuk and Shepherd 2001b).

Particle advection is carried out using the same method as for the PEM, although in this case the advection is over 30 days, and only the last 25 days are used for particle velocity calculations to allow for a period of initialization. Particle positions are sampled every $12 \mathrm{~h}$ as before, but only forward trajectories are used. Figure 11a shows the particle advection at day 30 for an adiabatic particle advection calculation on the $1000-\mathrm{K}$ isentropic surface. It is clear that although $m=2$ is the dominant wavenumber, smaller-scale waves are also evident everywhere. There are also waves in the summer (northern) hemisphere that appear to be smaller scale and transient. The surf zone here is also larger than in the PEM runs, extending from $50^{\circ}$ to $5^{\circ} \mathrm{S}$. Particles in the $\mathrm{NH}$ exhibit the presence of small-scale waves, but do not mix extensively. The presence of transient waves in the $\mathrm{NH}$ prevents this case from resembling the $\mathrm{SH}$ in the PEM runs (except for case B2).

Adiabatic transport calculations show similar results 

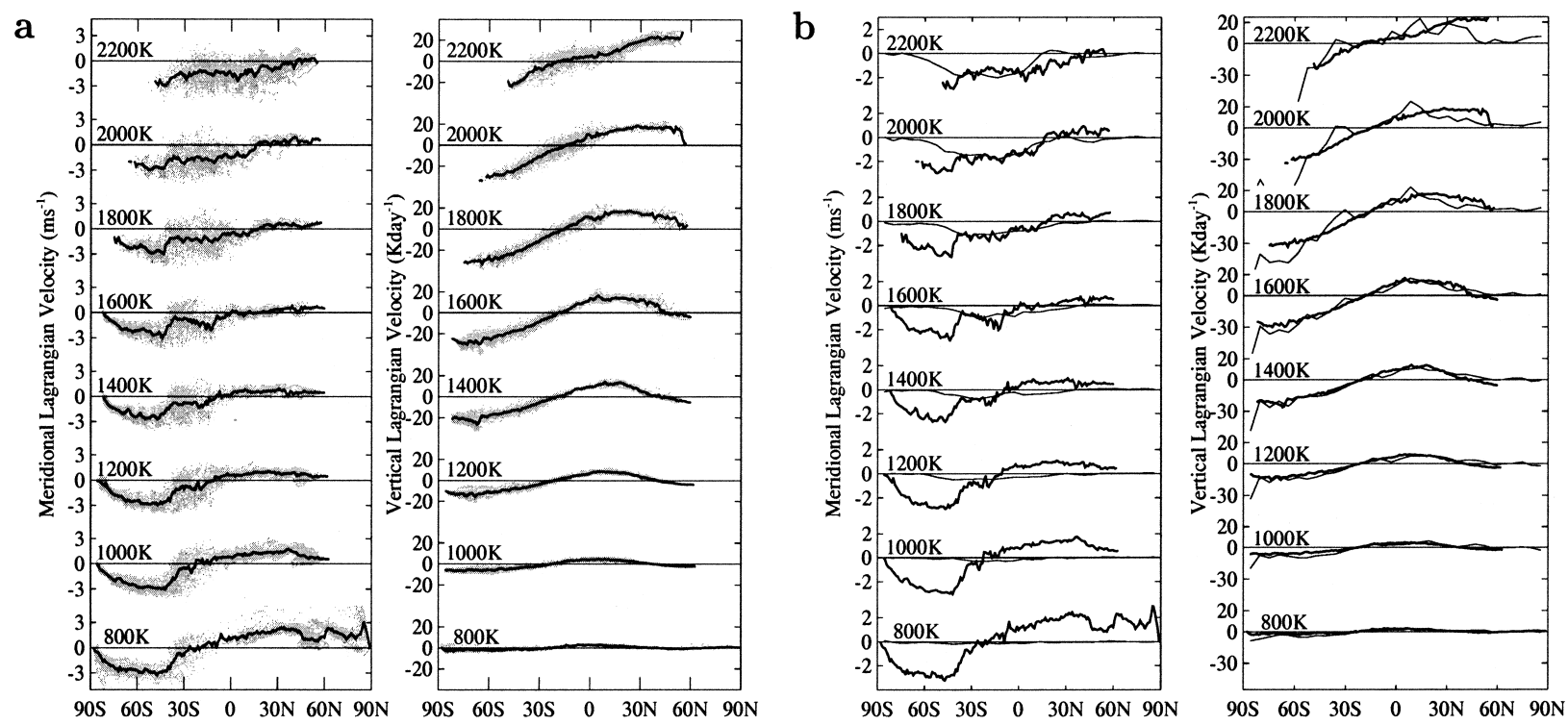

FIG. 12. (a) Meridional and vertical Lagrangian velocities for the CMAM run, using Lagrangian mean latitude and height as the particle labels. (b) Comparison of TEM (thin line) and Lagrangian (thick line) velocities.

to those from the PEM, although they are slightly less clear. Figure $11 \mathrm{~b}$ shows the meridional Lagrangian velocity using $\varphi_{L}$ as the particle label. Lagrangian velocities clearly show the surf zone, centered at $30^{\circ} \mathrm{S}$, with a minimum (but poleward) $\bar{v}_{L}$, and the two peaks in $\bar{v}_{L}$ closer to the surf zone edges. In this case, however, the peak located on the poleward edge of the surf zone indicates equatorward transport. Separate analysis shows that this is the result of entrainment of particles from the polar vortex into the surf zone during a particularly large wave-breaking event that occurs during July.

The diabatic transport calculations, shown in Fig. 12a, clearly show the Brewer-Dobson circulation, although in contrast to many of the PEM runs, diabatic dispersion occurs at all levels. Also, the double-peak structure seen in the adiabatic results and the PEM runs has disappeared. This suggests that the transport barriers that exist in the adiabatic transport calculations are not as strong when cross-isentropic motion is taken into account. It

TABLE 2. Approximate height of isentropes.

\begin{tabular}{ccc}
\hline & \multicolumn{2}{c}{ Approx height $(\mathrm{km})$} \\
\cline { 2 - 3 } Isentrope & PEM & CMAM \\
\hline 2200 & - & 52 \\
2000 & - & 49 \\
1800 & - & 47 \\
1600 & 56.5 & 44 \\
1400 & 53.5 & 41 \\
1200 & 50.0 & 39 \\
1000 & 45.0 & 35 \\
800 & 40.0 & 26 \\
600 & 32.8 & - \\
400 & 22.8 & - \\
\hline
\end{tabular}

should be noted that the background stratification is greater than that used in the PEM runs, so that model $\theta$ levels between the two sets of runs are not comparable (see Table 2).

The vertical TEM and Lagrangian velocities agree remarkably well, although not exactly so at higher $\theta$ levels where the TEM vertical velocities overestimate the descent in the polar vortex. (This is only clearly visible on the $1800-\mathrm{K}$ isentropic surface, since at 2000 and $2200 \mathrm{~K}$, particles inside the surf zone have descended below these levels.) The meridional TEM and Lagrangian velocities show much less agreement, and in some cases are not even of the same sign. There is particularly bad agreement at lower $\theta$ levels and in the polar vortex, where $\bar{v}_{L}$ is large compared with $\bar{v}^{*}$.

\section{Conclusions}

The current understanding of the Brewer-Dobson circulation is that planetary wave dissipation generally produces a negative EPFD in the stratosphere and therefore applies a negative (i.e., westward) torque on the stratospheric angular momentum. This negative torque produces a poleward mass flux, which through mass continuity produces the observed upwelling at the equator and downwelling at the pole. The TEM circulation is commonly used to describe the mean mass transport since it is composed of relatively easy-to-compute Eulerian quantities, and since under certain assumptions it is equivalent to the Lagrangian mean circulation. However, these assumptions are quite unrealistic in the stratosphere. In this study, the assumptions of linear, nonbreaking waves and Rayleigh friction have been relaxed. 
The focus of this paper is the quantitative relationship between the mean Lagrangian circulation and the TEM circulation. The classical theory (Dunkerton 1978) requires the flow to be quasigeostrophic, steady, conservative, and with small-amplitude waves, and all of these assumptions are questionable in the stratosphere. Even so, the vertical TEM and Lagrangian velocities from the PEM agree very well in regions of both nonbreaking waves and coherent stirring, where diabatic dispersion is not an issue. Diabatic dispersion becomes more important as the diabatic heating develops smaller horizontal scales, and as the stirring of particles becomes more chaotic. Nevertheless, the mean results agree remarkably well.

The meridional TEM and Lagrangian velocities agree well in regions of nonbreaking waves - that is, outside of the surf zone, where particles are restricted to undulating potential vorticity contours. The choice of particle label in this region also has little effect on the computed zonal-mean velocities. Within the surf zone, however, $\bar{v}^{*}$ and $\bar{v}_{L}$ do not agree as well. The meridional Lagrangian velocity is highly sensitive to the choice of particle label; using the Lagrangian-mean latitude $\varphi_{L}$ as the label, $\bar{v}_{L}$ reflects barriers between mixing and nonmixing regions, resulting in peaks near the barriers and a minimum in the mixing region where particles may be trapped for some time. The TEM velocity, on the other hand, suggests a uniform poleward transport through the surf zone, and does not reflect the kinematics of mixing.

The fact that $\bar{v}_{L}$ and $\bar{v}^{*}$ differ within the surf zone should not be interpreted as a failing of $\bar{v}^{*}$. Rather, it just means that within the surf zone the two fields are representing quite different aspects of the flow. Particle transport includes both a mean and a diffusive component. Within the surf zone, the diffusive component of the meridional motion is much greater than the mean component, and is highly spatially inhomogeneous. These spatial inhomogeneities determine the structure of $\bar{v}_{L}$. It is hardly surprising that under these circumstances $\bar{v}_{L}$ does not match $\bar{v}^{*}$, which only reflects the mean component of the motion; a symptom of this is that $\bar{v}_{L}$ does not satisfy a continuity equation with $\bar{w}_{L}$. In contrast, the mean component is much greater than the diffusive component for the meridional motion outside the surf zone, and for the vertical motion everywhere. That is why these features of the TEM and Lagrangian mean velocities are in such good quantitative agreement.

The fact that $\bar{w}_{L}$ and $\bar{w}^{*}$ agree so well, and that $\bar{w}^{*}$ and $\bar{v}^{*}$ satisfy a continuity equation, suggests that, if one could come up with a definition of a $\bar{v}_{L}$ that satisfied a continuity equation with $\bar{w}_{L}$, it would necessarily agree with $\bar{v}^{*}$. This possibility has not been pursued. On the other hand, the discrepancies between $\bar{v}_{L}$ and $\bar{v}^{*}$ within the surf zone might conceivably be attributed to the diffusive component of the particle motion as described in the TEM theory of transport (Andrews et al. 1987).
However, this theory is based on an assumption of small-amplitude disturbances, which is unlikely to be valid in the surf zone.

Acknowledgments. The authors would like to thank T. J. Dunkerton and an anonymous reviewer for their very helpful comments on the manuscript. Thanks are also due to Charles McLandress and David Sankey for providing the CMAM data. This work is based on DP's Ph.D. thesis, which was supported by the Natural Sciences and Engineering Research Council of Canada and the Ontario Graduate Scholarships program. TGS is supported by the Natural Sciences and Engineering Research Council of Canada, the Meteorological Service of Canada, and the Canadian Foundation for Climate and Atmospheric Sciences.

\section{REFERENCES}

Allen, D. R., and Coauthors, 1999: Observations of middle atmosphere CO from the UARS ISAMS during the early northern winter 1991/92. J. Atmos. Sci., 56, 563-583.

Andrews, D. G., 1983: A finite-amplitude Eliassen-Palm theorem in isentropic coordinates. J. Atmos. Sci., 40, 1877-1883.

_, and M. E. McIntyre, 1978: An exact theory of nonlinear waves on a Lagrangian-mean flow. J. Fluid Mech., 89, 609-646.

—_, J. R. Holton, and C. B. Leovy, 1987: Middle Atmosphere Dynamics. Academic Press, 489 pp.

Beagley, S. R., J. de Grandpré, J. N. Koshyk, N. A. McFarlane, and T. G. Shepherd, 1997: Radiative-dynamical climatology of the first-generation Canadian Middle Atmosphere Model. Atmos.Ocean, 35, 293-331.

Charney, J. G., and P. G. Drazin, 1961: Propagation of planetaryscale disturbances from the lower into the upper atmosphere. $J$. Geophys. Res., 66, 83-109.

Dritschel, D. G., 1989: Contour dynamics and contour surgery: Numerical algorithms for extended, high-resolution modelling of vortex dynamics in two-dimensional, inviscid, incompressible flows. Comput. Phys. Rep., 10, 77-146.

Dunkerton, T. J., 1978: On the mean meridional mass motions of the stratosphere and mesosphere. J. Atmos. Sci., 35, 2325-2333.

, 1989: Nonlinear Hadley circulation driven by asymmetric differential heating. J. Atmos. Sci., 46, 956-974.

_ 1991 : Nonlinear propagation of zonal winds in an atmosphere with Newtonian cooling and equatorial wavedriving. J. Atmos. Sci., 48, 236-263.

Edmon, H. J., B. J. Hoskins, and M. E. McIntyre, 1980: EliassenPalm cross sections for the troposphere. J. Atmos. Sci., 37, 26002616; Corrigendum, 38, 1115.

Fels, S. B., 1985: Radiative-dynamical interactions in the middle atmosphere. Adv. Geophys., 28A, 277-300.

Garcia, R. R., 1987: On the mean meridional circulation of the middle atmosphere. J. Atmos. Sci., 44, 3599-3609.

Haynes, P. H., and M. E. McIntyre, 1987: On the representation of Rossby wave critical layers and wave breaking in zonally truncated models. J. Atmos. Sci., 44, 2359-2382.

— , and E. Shuckburgh, 2000: Effective diffusivity as a diagnostic of atmospheric transport. 1. Stratosphere. J. Geophys. Res., 105, $22777-22794$.

_ C. C. Marks, M. E. McIntyre, T. G. Shepherd, and K. P. Shine, 1991: On the "downward control" of extratropical diabatic circulations by eddy-induced mean zonal forces. J. Atmos. Sci., 48, 651-678.

Kida, H., 1983: General circulation of air parcels and transport characteristics derived from a hemispheric GCM. Part 1. A deter- 
mination of advective mass flow in the lower stratosphere. $J$. Meteor. Soc. Japan, 61, 171-187.

McIntyre, M. E., 1980a: An introduction to the generalized Lagrangian-mean description of wave, mean-flow interaction. Pure Appl. Geophys., 118, 152-176.

__ 1980b: Towards a Lagrangian-mean description of stratospheric circulations and chemical transports. Philos. Trans. Roy. Soc. London, 296A, 129-148.

_ sphere. Nature, 305, 593-600.

Mo, R., and M. E. McIntyre, 1997: On anomalous meridional circulations and Eliassen-Palm flux divergences in an idealized model of dissipating, non-breaking Rossby waves. Dyn. Atmos. Oceans, 27, 575-600.

Nakamura, N., 1995: Modified Lagrangian-mean diagnostics of the stratospheric polar vortices. Part I: Formulation and analysis of GFDL SKYHI GCM. J. Atmos. Sci., 52, 2096-2108.

_ _ and J. Ma, 1997: Modified Lagrangian-mean diagnostics of the stratospheric polar vortices. 2. Nitrous oxide and seasonal barrier migration in the cryogenic limb array Etalon spectrometer and SKYHI general circulation model. J. Geophys. Res., 102, 25 72125735 .

Ngan, K., and T. G. Shepherd, 1999: A closer look at chaotic advection in the stratosphere. Part I: Geometric structure. J. Atmos. Sci., 56, 4134-4152.

Offermann, D., K.-U. Grossman, P. Barthol, P. Knieling, M. Riese, and R. Tran, 1999: Cryogenic infrared spectrometers and telescopes for the atmosphere (CRISTA) experiment and middle atmosphere variability. J. Geophys. Res., 104, 16 311-16 325.

O'Sullivan, D. J., and M. H. Hitchman, 1992: Inertial instability and Rossby wave breaking in a numerical model. J. Atmos. Sci., 49, 991-1002.

Plumb, R. A., and J. Eluszkiewicz, 1999: The Brewer-Dobson circulation: Dynamics of the tropical upwelling. J. Atmos. Sci., 56, $868-890$.

Randel, W. J., J. C. Gille, A. E. Roche, J. B. Kumer, J. L. Mergenthaler, J. W. Waters, E. F. Fishbein, and W. A. Lahoz, 1993:
Stratospheric transport from the tropics to middle latitudes by planetary-wave mixing. Nature, $\mathbf{3 6 5}, 533-535$.

_ , R. R. Garcia, and F. Wu, 2002: Time-dependent upwelling in the tropical lower stratosphere estimated from the zonal-mean momentum budget. J. Atmos. Sci., 59, 2141-2152.

Rhines, P. B., 1977: The dynamics of unsteady currents. The SeaIdeas and Observations on Progress in the Study of the Seas, E. D. Goldberg et al., Eds., Marine Modeling, Vol. 6, John Wiley and Sons, $189-318$.

Rood, R. B., and M. R. Schoeberl, 1983: A mechanistic model of Eulerian, Lagrangian-mean and Lagrangian ozone transport by steady planetary waves. J. Geophys. Res., 88, 5208-5218.

Rosenlof, K. H., 1995: The seasonal cycle of the residual mean meridional circulation in the stratosphere. J. Geophys. Res., 100, 5173-5191.

Schoeberl, M. R., 1981: A simple model of the Lagrangian-mean flow produced by dissipating planetary waves. J. Atmos. Sci., 38, 1841-1855.

Scott, R. K., and P. H. Haynes, 2000: Internal vacillations in stratosphere-only models. J. Atmos. Sci., 57, 3233-3250.

Semeniuk, K., and T. G. Shepherd, 2001a: Mechanisms for tropical upwelling in the stratosphere. J. Atmos. Sci., 58, 3097-3115.

— , and — 2001b: The middle-atmosphere Hadley circulation and equatorial inertial adjustment. J. Atmos. Sci., 58, 3077-3096.

Shepherd, T. G., 1987: Rossby waves and two-dimensional turbulence in a large-scale zonal jet. J. Fluid Mech., 183, 467-509.

_ 2000: The middle atmosphere. J. Atmos. Sol.-Terr. Phys., 62, $1587-1601$.

_ 2002: Issues in stratosphere-troposphere coupling. J. Meteor. Soc. Japan, 80, 769-792.

_ K. Kemeniuk, and J. N. Koshyk, 1996: Sponge layer feedbacks in middle-atmosphere models. J. Geophys. Res., 101, $23447-$ 23464.

_ J. J. N. Koshyk, and K. Ngan, 2000: On the nature of large-scale mixing in the stratosphere and mesosphere. J. Geophys. Res., 105, 12 433-12 446.

Sparling, L. C., J. A. Kettleborough, P. H. Haynes, M. E. McIntyre, J. E. Rosenfield, M. R. Schoeberl, and P. A. Newman, 1997: Diabatic cross-isentropic dispersion in the lower stratosphere. $J$. Geophys. Res., 102, 25 817-25 829. 